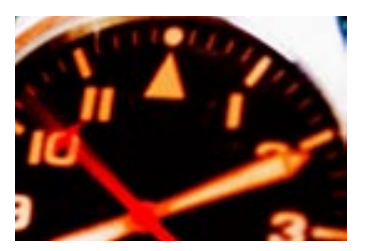

10-MINUTE CONSULTATION

Newly diagnosed iron deficiency anaemia in a premenopausal woman

\author{
Tony Todd, ${ }^{1}$ Tim Caroe $^{2}$
}

'Addenbrooke's Hospital, Cambridge CB2 2QQ

${ }^{2}$ General Practice and Primary

Care Research Unit, University of

Cambridge

Correspondence to: T Todd

tony.todd@addenbrookes.nhs.uk

BMJ 2007:334:259

doi:10.1136/bmj.39003.602338.94

\section{USEFUL READING}

\section{British Society of} Gastroenterology. Guidelines for the management of iron deficiency anaemia. www. bsg.org.uk/pdf_word docs/iron_def.pdf NHS National Library for Health. Iron deficiency anaemia. www.prodigy.nhs.uk/ patient_information/pils/ anaemia_due_to_iron_ deficiency.htm (a helpful information leaflet for patients)
A 40 year old woman with persistent fatigue has come back to you for the results of blood tests. The results show a hypochromic, microcytic anaemia with a haemoglobin concentration of $100 \mathrm{~g} / \mathrm{l}$ and a ferritin concentration of $5 \mu \mathrm{g} / \mathrm{l}$, the classic features of iron deficiency anaemia (IDA).

\section{What issues you should cover \\ Symptoms of anaemia}

Fatigue and mild dyspnoea after exertion may be the only symptoms in otherwise healthy people with slow onset anaemia. More serious symptoms such as angina, marked ankle oedema, or dyspnoea at rest don't generally occur unless the haemoglobin concentration is less than about $70 \mathrm{~g} / \mathrm{l}$. Presence of such symptoms in a case like this indicates additional cardiorespiratory pathology.

\section{Causes of the anaemia}

Blood loss-Seek any history of haemorrhage. In the $5-10 \%$ of premenopausal women who develop IDA the commonest cause is menorrhagia. For reliable assessment simple pictogram charts may be better than subjective reporting.

Coeliac disease-In younger patients coeliac disease often presents with IDA, especially in women aged 20-40. Pay attention to gastrointestinal symptoms, including unexplained weight loss, diarrhoea, and abdominal pain. Drug use-Some drugs, such as anticoagulants, aspirin, and non-steroidal anti-inflammatory drugs, may cause or exacerbate blood loss. Warfarin may worsen menstrual bleeding but rarely causes other major chronic haemorrhage (such as gut or genitourinary bleeding) without additional pathology. Warfarin may, therefore, be unmasking another pathology, and you should still seek an underlying cause of IDA.

Other history-Consider recent pregnancy, breast feeding, and blood donation. Pregnancy induced IDA usually resolves rapidly after birth but may persist in women from low income groups who have a poor diet, especially if they are breast feeding. Frequency of blood donation is limited to prevent IDA, but it may still occur, as in premenopausal women each unit contains about a tenth of the average amount of body iron.

Diet-In the United Kingdom IDA unrelated to pregnancy is rarely caused by poor diet, as major deficiency would arise only after several years without any food that contains iron (meat, leafy green vegetables).

\section{Features of iron deficiency}

Some classic features of IDA-koilonychia, angular stomatitis, and glossitis-are rare but distressing. They resolve on treatment.

\section{Key points}

- Iron deficiency is not a final diagnosis-you must identify and treat the underlying causes

- In premenopausal women, particularly consider menorrhagia (a simple visual scoring system may help in assessment) and coeliac disease

- Poor diet and warfarin use are rarely sole causes

- A significant response should occur within three weeks of treatment starting

\section{What you should do}

- No degree of IDA should be ignored. Audits show that iron deficiency is underinvestigated and that serious illness can be missed. Even when a cause seems clear, failure to respond to treatment should raise suspicion of alternative causes.

- Refer appropriately in cases of abnormal blood loss. Referral to gastroenterology is indicated if she has gastrointestinal symptoms, obvious gastrointestinal blood loss, or a strong family history of colorectal carcinoma (such as two first degree relatives or one first degree relative aged $<45$ years). Gastrointestinal referral is not needed in asymptomatic women under 50 unless they don't respond to adequate iron supplementation and treatment of other identified causes. In women $4 \%$ of cases of IDA are due to coeliac disease, which may otherwise be asymptomatic. Women with relevant symptoms, those whose IDA lacks an apparent cause, and those who don't respond to iron treatment should be tested for anti-endomysial or anti-transglutaminase antibodies. Faecal occult blood testing is unpleasant and of little diagnostic value.

- Treat identified underlying causes. Start replacement treatment with ferrous sulphate $200 \mathrm{mg}$ three times a day (ferrous gluconate and ferrous fumarate are alternatives). Continue for three months after the anaemia resolves to fully replenish stores. Avoid slow release preparations, as these release most of their iron after passing the small bowel absorption site.

- Warn her of side effects of taking iron (constipation and black stool) and stress the importance of adherence. Also explain that IDA is a consequence of something else that iron alone won't treat and that this needs proper investigation and treatment.

- Arrange a follow-up full blood count and consultation in three weeks. A rise of haemoglobin by then of $<20 \mathrm{~g} / \mathrm{l}$ indicates a problem with adherence, malabsorption, or continuing blood loss.

This is part of a series of occasional articles on common problems in primary care

The $B M$ / welcomes contributions from general practitioners to the series 